\title{
Recognition for Organometallic Chemistry
}

\section{Professor P. L. Pauson of the University of Strathclyde summarises the work on organo-transition metal chem- istry which has earned its two chief founders the 1973 Nobel Prize for Chemistry.}

ERNST OTTo Fischer and Geoffrey Wilkinson share this year's Nobel Prize for Chemistry for spearheading the spectacular advances in organo-transition metal chemistry during the past twenty years. Not only were they among the first to recognise in 1952 the unique structure of ferrocene but their success in synthesising other "metallocenes" and many related cyclopentadienyl-metal complexes opened up the whole field.

\section{Sandwich Compounds}

Wilkinson used the name "sandwich compounds" for the metallocenes to describe molecular architecture, a structure consisting of two parallel fivemembered carbon rings (cyclopentadienyl groups) symmetrically arranged around the central metal atom. The rings may be regarded as having sextets of $\pi$ electrons and the important consequence, the aromaticity of ferrocene, was recognised and demonstrated by $\mathbf{R}$. B. Woodward. The extensive organic chemistry thus opened up continues to be widely explored, most intensively and successfully in A. N. Nesmeyanov's Moscow laboratory, whose recent work has revived interest in ferrocene derivatives as anti-anaemic agents. Despite some early commercial promise, for example, for vapour plating and as fuel additives-including anti-knock agents, where a cyclopentadienylmanganese compound has earned limited practical success-no major direct application has yet repaid the intense effort put into this field. Initially industrial interest was so strong that it was not uncommon for a new type of cyclopentadienyl complex to be synthesised independently in three industrial laboratories as well as in Fischer's and Wilkinson's laboratories. But the work of Fischer and Wilkinson gradually diverged as the field broadened.

Theoretical interest in these remarkable structures was no less intense than practical interest, and gradually deepening understanding helped greatly in extending the whole area of research. J. Chatt and L. A. Duncanson had taken an important step in 1953 by applying
M. J. S. Dewar's $\pi$-bonding ideas to explain the structure of the organometallic compound known longer than any other-Zeise's ethylene-platinum salt. The relationship to this and the cyclopentadienyl sandwiches of other known compounds, including some acetylene complexes first prepared by W. Reppe and his group as well as $\mathbf{H}$. Reihlen's butadiene-iron complex, emerged gradually. During this period both Fischer and Wilkinson worked on a variety of related alkene, diene and triene complexes.

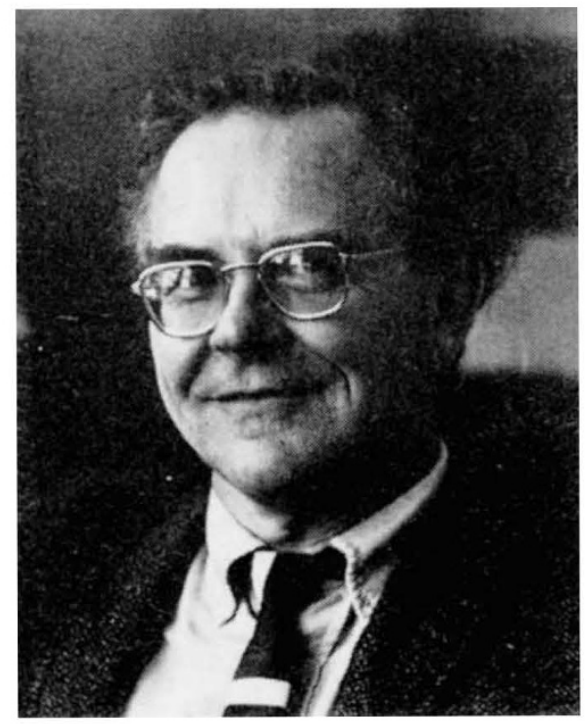

Professor Geoffrey Wilkinson

Suspicion that the most puzzling of the earlier discoveries, F. Hein's "polyphenylchromium" compounds, might also belong in the "sandwich" class received striking confirmation when $\mathbf{E}$. $\mathbf{O}$. Fischer and W. Hafner succeeded in synthesising the prototype: bisbenzenechromium. With the impressive range of other arene-metal complexes subsequently synthesised in his laboratory, this will undoubtedly continue to be regarded as Fischer's most distinctive and important contribution.

More recently, most of his work has been concentrated on the intriguing carbene complexes, first prepared in his laboratory as a result of a most skilful and purposeful search for suitable synthetic methods. These studies have taken him back much closer to the metal carbonyls, on which he worked as a student under W. Hieber, whom he succeeded in the chair of inorganic chemistry at the Technische Hochschule of Munich in 1964.

Fischer's entire scientific work to date has been done there and at the University of Munich (1957-64). He resisted calls to other chairs which would have taken him from his native city and further from the Alps which he loves and where he relaxes-usually in the company of his students, walking and skiing from an attractive chalet built with the proceeds of the bis-arenechromium patents.

\section{Return to Imperial College}

Wilkinson-a Yorkshireman born in Todmorden-likewise holds office in his own alma mater, the Imperial College of Science and Technology, London. He returned there in 1956 after thirteen years in North America, having gone to Canada during the war to do radiochemical work in connection with the development of the atomic bomb and moving by way of California to the Massachusetts Institute of Technology and then Harvard.

Most of his "sandwich" work was carried out at Harvard where Al Cotton, co-author of his famous textbook, was the first student to work with him in this field. Among Wilkinson's contributions of that period, his use of the then quite new NMR techniques demonstrated their great potential in this field and led to later discoveries of many interesting aspects of the behaviour of organometallic molecules. Since returning to London, his interests have gradually moved to other areas, notably the transition metal alkyls (he has devised molecules of almost as surprising stability as the original "sandwiches") and catalysis (he found and studied some of the most active homogeneous hydrogenation catalysts).

It is in such catalytic aspects. and in the use as chemical intermediates of such metal complexes as Fischer's carbene derivatives, that greatest interest and promise of use resides at present; but regardless of future developments, this year's Nobel Laureates have added a remarkable chapter to chemical knowledge. 